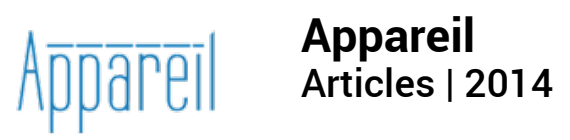

\title{
Matérialité nombreuse et spiritualité sans fond dans Les Soprano
}

\section{Aymen Gharbi}

\section{OpenEdition}

Journals

Édition électronique

URL : http://journals.openedition.org/appareil/2043

DOI : 10.4000/appareil.2043

ISSN : 2101-0714

\section{Éditeur}

MSH Paris Nord

\section{Référence électronique}

Aymen Gharbi, «Matérialité nombreuse et spiritualité sans fond dans Les Soprano », Appareil [En ligne], Articles, mis en ligne le 28 août 2014, consulté le 30 juillet 2020. URL : http://journals.openedition.org/ appareil/2043 ; DOI : https://doi.org/10.4000/appareil.2043

Ce document a été généré automatiquement le 30 juillet 2020.

\section{(c) (i) (9)}

Appareil est mis à disposition selon les termes de la Licence Creative Commons Attribution - Pas d'Utilisation Commerciale - Pas de Modification 4.0 International. 


\title{
Matérialité nombreuse et spiritualité sans fond dans Les Soprano
}

\author{
Aymen Gharbi
}

\section{Introduction}

1 Un parrain de la mafia se rend chez sa psychanalyste, dans le dessein de lui exposer ses déboires psychologiques et physiques. Une peinture de plus en plus détaillée de son univers mental, familial et professionnel, qu'il contrôlera au début mais au cours de laquelle il perdra son statut de narrateur homodiégétique ${ }^{1}$, va se déployer en quatrevingt-six épisodes d'environ une heure chacun. Le premier épisode des Soprano doit être considéré comme l'exposition d'une problématique : le mafieux Tony Soprano, à la tête d'une armée de soldats ${ }^{2}$, issu d'une lignée spéciale dont la série porte le nom, se trouve confronté à la béance originelle qui ne cessera de l'obnubiler et qui cristallise une somatisation de son trouble: une syncope, une lourde chute dans les abîmes obscurs de l'inconscient crée la rupture originelle catalylisatrice de la narration ${ }^{3}$. Ou comment ce corps issu de la geste épique de l'immigration de la vieille Europe vers l'Amérique, vertical et viril, déployé en un espace de conquête, suivant à grande vitesse une trajectoire vers l'enrichissement matériel dans un environnement propice à la consommation effrénée, s'effondre-il ? Comment passe-il à une horizontalité annonciatrice de ses sommeils cauchemardesques à venir, de l'état comateux proche de la mort qu'il vivra à la $\mathrm{VI}^{\mathrm{e}}$ saison, ainsi que d'un terrassement dépressif global, personnifiant une époque comme disait Émile Zola à propos des Rougon-Macquart ?

2 Cet évanouissement est problématique dans le sens où il place un personnage central peu habitué à l'abstraction en face d'un programme d'ordre herméneutique. Il s'agit pour lui d'œuvrer à sonder son inconscient énigmatique et subversif alors qu'il en est empêché par un mouvement sémiotiquement opposant : à l'aide du travail accoucheur ${ }^{4}$ de sa psychanalyste, Tony devra dire ce qui doit être tu dans un milieu mafieux 
anthropologiquement pérennisé par le non-dit. Il devra atténuer sa culpabilité cinglante bien qu'il soit le chef d'une organisation où le meurtre de sang-froid est une coutume coercitive. Il devra se pencher sur l'immatérialité confuse de ce que Cornélius Castoriadis nomme allégoriquement l'Abîme/Chaos/Sans-Fond, lorsqu'il est happé par la matérialité foisonnante d'un milieu (prépondérance de la valeur d'échange dans la mafia) et plus largement de tout un système économique (prépondérance de la valeur d'échange aux États-Unis).

\section{« On mange beaucoup... »}

3 Les syncopes réitérées de Tony dégonflent son corps par un effet mécanique et font valoir régulièrement que l'énigme herméneutique ${ }^{5}$ initiale reste à résoudre tant qu'il $^{\prime}$ n'arrive pas à donner une quelconque consistance structurante à sa boulimie nutritive. $\mathrm{Au}$ contraire, le poids qu'il prend en se nourrissant de manière effrénée lui attire les moqueries en catimini de son entourage et de dangereux problèmes de santé. Il faut dire que la mangeaille est omniprésente, gonflant sensuellement l'image, surtout dès que Tony apparaît. Innombrables sont les plans et les séquences où nous voyons des personnages dialoguer tout en avalant des sandwiches fast-food, des plats de restaurant, des déjeuners de famille, des apéritifs de réceptions, des dîners mondains, des petits déjeuners copieux. La boisson, jus, alcool, lait, etc. est tout aussi présente. Et innombrables sont les moments où Tony dévore symboliquement son interlocuteur lors de froides négociations professionnelles ou bien lors de conflits de famille castrateurs, autour d'un bon plat ${ }^{6}$. Au cours du huitième épisode de la III $^{\mathrm{e}}$ saison, lorsqu'il veut entendre les excuses de Ralph Cifaretto à propos d'un affront, il l'humilie au cours d'une cérémonie de domination dans le restaurant Le Vesuvio, en pleine ripaille. L'esprit de Tony pense aux sauces avec lesquelles il dégustera le monde ainsi qu'aux différents commensaux hostiles qui le composent. Ainsi, un parti pris plastique favorisant le contact charnel avec la nourriture et la boisson, met sa bouche en phase de mastication sans trêve ni repos. Elle fonctionne à plein régime, effectuant un travail de mâchage compulsif pour remplir le ventre. Elle réduit le dur en amorphe et tout se passe comme si, à défaut d'articuler un discours libérateur qui installerait un début d' autonomie $^{7}$ grâce à la thérapie psychanalytique ou à l'aveu sincère, cette bouche ne pouvait que réduire en miette les composantes d'une matérialité frustrante. En plus d'être le signe généralisé d'une compensation boulimique d'un manque, ce mode de représentation de l'ingurgitation est la métaphore d'un caractère carnassier qui ne recule devant aucun crime pour prospérer et qui n'hésite devant aucun broyage de cadavre pour effacer matériellement les signes oppressants de sa culpabilité.

C'est cette insatiabilité étrange qui rend le ventre de Tony aussi proéminent, ventre qu'il contemple souvent en privé, non sans éprouver un certain ahurissement. Notamment lorsqu'il est, au deuxième épisode de la $V^{e}$ saison, seul et saoul au milieu du vide nocturne de la maison de sa mère décédée. Dans cette scène où il lorgne son reflet dans un miroir, après avoir fait un cauchemar, pèse la lourde saillance de sa panse qu'il palpe comme s'il avait envie de l'ouvrir pour y ausculter une indicible culpabilité qui s'apparente en l'occurrence à un inconsolable vagissement. Ne dit-il pas, à un certain moment, à un médecin qu'il préférerait que son mal soit physique car dans ce cas au moins ses signes seraient plus limpides que les sombres circonvolutions œdipiennes? 
$5 \mathrm{Au}$ début de la $\mathrm{VI}^{\mathrm{e}}$ saison, une plaie béante en forme de cercle s'ouvre dans ce même ventre, lorsqu'il entre dans le coma après que son oncle, Corrado Soprano, ait tiré sur lui. La béance est plus que jamais grande avec cette perforation affolante du pancréas, organe participant à la digestion, qui offre le spectacle d'un fantastique cronenbergien ${ }^{8}$ à la vue de ses visiteurs, tandis que sa vie semble s'en échapper. Ce trou a l'air d'être l'allégorie ${ }^{9}$ même de l'idée de l'Abîme/Chaos/Sans-Fond: ce magma métaphysique castoridien qui se régénère indéfiniment depuis les profondeurs conscientes et inconscientes de la psyché humaine et qui génère, au niveau collectif, les institutions et les symboles. La fonction principale de l'art, selon Castoriadis, outre le fait qu'il provient de cet Abîme/Chaos/Sans-Fond, serait de donner « directement, sur le chaos $»^{10}$. Quoi de plus direct, en l'occurrence, que ce fascinant trou sphérique et purulent qui s'ouvre dans «les tripes" de Tony, comme on dit dans un registre familier pour désigner la profondeur de l'âme humaine?

6 L'association entre remplissage et évanouissement n'est jamais littérale. Seulement dans le premier épisode de la $\mathrm{V}^{\mathrm{e}}$ saison, l'écart sous-jacent entre ces deux éléments se trouve totalement réduit lorsque le double de Tony, autre parrain qu'on voit également manger sans cesse, Carmine, meurt. Pendant un déjeuner avec Tony notamment, il étouffe d'un trop-plein non assimilé qui déborde par une crise cardiaque fatale, alors que sa bouche est gavée de nourriture, comme si la machine dévorante ne fonctionnait plus.

7 Pour faire le lien entre digestion et états d'âmes dans une perspective esthétique, l'on pourrait dire en paraphrasant l'analyse bachelardienne que fait Jean-Pierre Richard de la littérature de Gustave Flaubert, qu'on mange beaucoup ${ }^{11}$ dans Les Soprano. Et l'on pourrait aussi ajouter qu'on y digère beaucoup, parfois jusqu'à l'épuisement. Ce même Flaubert est d'ailleurs cité au deuxième épisode de la $\mathrm{V}^{\mathrm{e}}$ saison par le professeur du fils de Carmela, au cours d'un déjeuner avec elle où il lui recommande la lecture d'un de ses romans. Afin de mieux la confronter à sa condition de femme au foyer délaissée, il lui tend le miroir de Madame Bovary pour qu'elle s'y mire. Mais il ne serait pas exagéré de dire qu'à un niveau diégétique, c'est plutôt la mécanique narrative qui attelle Carmela à la lecture de Flaubert afin qu'elle en infère le drame implicite de son propre univers. La définition de l'esthétique proposée par Jacques Rancière jette une lumière sur cette tension réflexive conduisant Carmela à projeter ce roman sur sa propre vie :

Esthétique, pour moi, désigne un mode de pensée qui se déploie à propos des choses de l'art et s'attache à dire en quoi elles sont des choses de pensée. Plus fondamentalement, c'est un régime historique spécifique de pensée de l'art, une idée de la pensée selon laquelle les choses de l'art sont des choses de pensée. ${ }^{12}$

Ce drame implicite, à la lisière de l'inconscient, qui régit son propre univers implique cette même gloutonnerie flaubertienne, boulimique, aux ramifications multidimensionnelles identifiée par Jean-Pierre Richard, où "l'appétit se fait hystérique, déchainé $»^{13}$ et où, par la force des choses, la digestion inclut des contractions problématiques.

\section{L'individu problématique dans la culture de masse}

9 La gloutonnerie est d'autant plus éloquente que lorsqu'il ouvre le réfrigérateur ou lorsqu'il est au restaurant, topoï importants et récurrents dans l'œuvre, Tony est confronté à une variété de marques de plats et de boissons. De façon générale, les 
personnages souvent conversent non seulement dans ces deux espaces mais aussi dans des hypermarchés et des centres commerciaux, entre deux achats ou entre deux surveillances policières. Or leur navigation au milieu de la variété quantitative dont ils ont l'air de s'enorgueillir, n'est pas anodine : pour une fois ces individus qui étouffent existentiellement se sentent libres... de consommer. C'est schématiquement ce que certains philosophes ont diagnostiqué comme la maladie sénile de l'individualisme : la liberté tant chantée de l'Homme est tributaire du système économique et le libéralisme, qui était au départ une doctrine philosophique prônant la conservation de l'individu dans une société dont les soubassements moraux hérités des guerres civiles moyenâgeuses ont commencé à être démontés avec les Lumières, devient, comme l'ont montré les analyses de Jean-Claude Michéa, une nouvelle forme de violence conduisant à la dictature impersonnelle de la consommation et du spectacle ${ }^{14}$.

10 Ainsi l'on peut tisser le lien entre Flaubert, Les Soprano et ce que Lucien Goldmann, dans une approche marxiste et parfois trop étroite de la genèse du genre romanesque, nomme l'individu problématique ${ }^{15}$. Cet individu fictionnel propulsé par l'entrée du roman dans l'histoire littéraire, concomitamment au développement du système économique capitaliste, prend naissance de « la contradiction interne entre l'individualisme comme valeur universelle engendrée par la société bourgeoise et les limitations importantes et pénibles que cette société apportait en réalité elle-même aux possibilités de développement des individus ${ }^{16}$.

11 Les manifestations de cette contradiction interne sont si prégnantes dans notre œuvre qu'on ne peut ignorer une interprétation marxiste et postmarxiste, non sans l'enrichir par des paradigmes pertinents comme la théorie de la réception de Hans Robert Jauss ${ }^{17}$. Car si Lucien Goldmann est arrivé à la certitude historiciste que le roman est né au sein de l'individualisme, il faudrait ajouter qu'au niveau de sa communication au récepteur, il a prospéré, contrairement au genre tragique et épique, entre autres grâce à la presse écrite, média de masse aussi péjorativement considéré par les «intellectuels » de l'époque que ne l'est la télévision aujourd'hui. L'émergence dans l'histoire de la télévision de la série moderne, entamée à la fin des années 1990, pourrait être étudiée à la lumière du télescopage du roman avec la presse écrite au XIX $x^{e}$ siècle.

12 En effet, aussi bien des écrivains comme Théophile Gautier ${ }^{18}$ ou Balzac ${ }^{19}$ que des sociologues comme Gabriel Tarde ${ }^{20}$, soupçonnaient les journaux d'appauvrir la pensée, de mercantiliser le savoir et de manipuler idéologiquement le public, ce qu'on peut mettre en parallèle avec la posture actuelle de certains artistes ${ }^{21}$ et intellectuels devant la télévision ${ }^{22}$. On pourrait d'ailleurs faire dialoguer Gabriel Tarde, s'attelant à dégager les effets criminogènes de la rubrique des faits divers dans les journaux ${ }^{23}$, avec certains chercheurs contemporains liant le sensationnalisme et la violence télévisuelle à la criminalité des jeunes et même à l'embrigadement terroriste. L'articulation historique où la littérature bascule vers une démocratisation industrielle menant à la culture de masse dont le cinéma et la télévision deviendront les parangons ${ }^{24}$, est bien là, esquissée par les balbutiements mercantiles des romans-feuilletons, comme le constate Arnold Hauser lorsqu'il affirme que l'œuvre littéraire au XIXe siècle est devenue « un "objet usuel", au sens le plus strict du terme. Elle est tarifée, produite conformément à une norme acceptée et livrée dans des délais fixés à l'avance ${ }^{25}$. Et dans cette perspective nous renvoyons aussi au travail d'Antoine Compagnon sur la modernité dans Les cinq paradoxes de la modernité. Parmi les cinq paradoxes esthétiques fondamentaux qu'il pose, nous trouvons l'appel à la culture de masse qui est cette « domination croissante du 
marché sur l'art et sur la littérature » et « leur médiatisation de plus en plus poussée ».

Néanmoins, nous pouvons dire que ce genre de paradoxe, malgré ses inconvénients, n’a pas empêché Gautier et Balzac de s'acclimater à la presse par des œuvres telles que Fortunio et La Vieille Fille. Elle n'a pas non plus empêché le fait que Les Soprano, diffusée en 1999 sur la chaîne de télévision américaine HBO, soit une série dans laquelle et à travers laquelle la production télévisuelle est devenue perméable à l'art et à la littérature, tout comme les journaux le furent, pendant un temps, aux grands textes littéraires publiés sous la forme de romans-feuilletons. Surdéterminée par une intertextualité massive, une capacité à renouer avec l'ampleur vertigineuse des grands individus problématiques de la littérature romanesque et une maîtrise de la grammaire cinématographique qui rompt avec la platitude télévisuelle, notre série a la prétention patente de construire des ponts avec l'histoire de la pensée et de l'art. Cela dit, elle s'y applique tout en usant du système figuratif d'un média caractérisé par une inauthenticité intrinsèque, si l'on en croit Adorno et Horkheimer qui désignent sous la fameuse formule de l'industrie culturell ${ }^{27}$ cet art moderne qui condamne sa poétique à succomber à l'appel de l'industrie ${ }^{28}$.

14 À l'image du roman dont les individus problématiques ont triomphé en même temps que l'avènement d'un monde individualiste venu s'installer sur les décombres de la vieille féodalité, la série télé pourrait s'inscrire dans un irrépressible mouvement de mutation de l'art et de la littérature à l'aire de l'économie de marché. Sans doute le point commun entre ces périodes de l'histoire, nonobstant le canal de communication, serait la faculté d'une œuvre de voir cette obscurité dont parle Giorgio Agamben lorsqu'il affirme que " seul peut se dire contemporain celui qui ne se laisse pas aveugler par les lumières du siècle et parvient à saisir en elles la part de l'ombre, leur sombre intimité » 29 .

15 Quoi qu'il en soit, industrielle et artistique, spectaculaire et littéraire, lumineuse et obscure, Les Soprano est une œuvre globalement polarisée autour de tendances que d'aucuns qualifieraient d'irréconciliables. Elle est ainsi à l'instar de ses personnages perdus entre le dicible et l'indicible, doutant d'eux-mêmes et du monde qui les entoure ; et incapables même d'atteindre cet état d'apathie qu'évoque Tocqueville dans De la démocratie en Amérique, en s'attachant « uniquement aux instincts et aux intérêts matériels, qui sont bien plus visibles, plus saisissables et plus permanents de leur nature que les opinions $»^{30}$.

\section{Le paradoxe écrasant}

Innombrables donc sont les scènes où l'on mange. Mais comme s'il s'agissait de découvrir l'envers de cette vitrine salubre et alléchante, innombrables sont les scènes où l'on se vide.

17 Adriana, happée par la béance lorsqu'elle a été acculée à devenir indicatrice du FBI, n'arrive plus à gérer sa boulimie moins attisée par la mangeaille que par l'euphorie de la drogue et celle des marques de luxe. Son angoisse est générée par le fait qu'un étau sociopolitique se resserre sur elle, américaine moyenne dépourvue d'un logos émancipateur qui lui permette de déconstruire l'imbroglio où elle s'est trouvée engluée : d'un côté la police, investissant beaucoup d'argent dans la lutte contre la mafia, la harcèle obstinément pour qu'elle lui livre des informations rentables qu'elle 
n'a pas. De l'autre côté son compagnon, le mafieux Christopher Moltisanti qui se proclame soldat de la mafia, amoureux violent et possessif, oppressé par le désir de réussite sociale et auquel elle est subordonnée économiquement. À partir de ce point de non-retour tragique où rien ne peut plus être révocable, elle est sans cesse prise de diarrhées et de nausées. Au deuxième épisode de la IV saison, une scène d'un réalisme très explicite qui ne peut que témoigner de la volonté de l'auteur de surligner cette incontinence anale et orale, la montre soudain vomir sur les agents du FBI qui la faisaient chanter dans le dessein de la forcer à coopérer. Ici ce n'est plus l'évanouissement qui est en jeu mais plutôt une incapacité à se retenir lorsque la béance creusée par ces policiers d'une froideur volontairement montrée comme inhumaine, tord l'anatomie. Les muscles du système digestif sont rompus par l'écrasement douloureux de la contradiction. La figure de l'allégorie est ici quintessenciée d'autant plus que la sinuosité de la matière des déglutitions qui s'étalent sur les policiers est plastiquement bien mise en relief par la mise en scène.

$18 \mathrm{Au}$ cinquième épisode de la $\mathrm{VI}^{\mathrm{e}}$ saison, Tony, en convalescence après que les médecins aient recousu la plaie béante de son ventre, veut imposer son leadership à ses hommes qui ont commencé à discerner sa faiblesse suite à son coma. Il faut signaler avant de poursuivre l'analyse de cette scène, que malgré son ethos de parrain bien manifesté, ses subalternes n'hésitent pas à lui montrer d'une façon implicite et explicite, que son règne est fragile, aussi fragile que celui d'un chef politique élu. Dans cette dialectique entre dominants et dominés, ils jouent parfois le rôle de son surmoi communautaire lorsqu'il s'agit de prendre des décisions punitives fermes contre ceux qui ont outrepassé les codes de la communauté mafieuse. Les rumeurs malveillantes sur sa psychanalyse, l'épisode de Tony B. son cousin transgressif, ou celui de l'homosexualité de Vito Spatafore, montrent qu'une pression collective aveugle, parfois hostile, s'exerce sur lui pour qu'il prenne la décision imposée par le dogme ancestral de l'organisation criminelle. L'étau qui se resserre sur lui dans cette conjonction, et qu'un développement de Michel Meyer sur le bouc émissaire tragique pourrait éclairer ${ }^{31}$, est celui de la réflexion sur des problèmes éthiques singuliers d'un côté et celui, lapidaire, de l'irréfragable dogme, de l'autre. Sentant donc que ses hommes le voient d'un autre œil après son hospitalisation, il tabasse gratuitement l'un d'entre eux, puis s'empresse de se réfugier dans les toilettes où il vomira du sang en abondance. Le circuit mental qui l'a conduit à ouvrir la porte de cette démesure arrogante contre l'un de ses hommes en le rossant, n'a pu être parcouru sans que son corps soit éviscéré par les lames du paradoxe.

Pareillement, au début de la $\mathrm{II}^{\mathrm{e}}$ saison, une intoxication alimentaire non seulement provoquera en lui des vomissements et une diarrhée mais sera le moteur d'un pullulement en chaîne de cauchemars. Ces cauchemars constitueront un sens sémiotique grâce auquel Tony sera convaincu qu'il faut assassiner Pussy qu'il soupçonnait sans preuves d'être un indicateur ${ }^{32}$. Le corps comme l'esprit ne sont plus étanches à une circulation torrentielle de matières et de signes inconscients, rhétoriquement convaincants. En d'autres termes, c'est la conviction de la culpabilité de son «meilleur ami » Pussy qui doit être prise en considération pour comprendre cet épisode tragicomique centré sur sa gastro-entérite. Au-delà de l'intoxication, l'étalement du contenu du ventre vient du dilemme pressant entre tuer son meilleur ami traitre pour des motivations dogmatiques et l'épargner pour des motivations éthiques. 
20 Vito Spatafore est la manifestation du marginal torturé par sa déviante différence par rapport aux normes de la communauté. Capitaine mafieux, il est secrètement homosexuel. Ce secret non dévoilé, qui lui fait encourir la peine de mort selon la loi de la communauté mafieuse, se manifeste involontairement sous la forme de son aérophagie et des blagues scatologiques de Tony sur la corpulence de ses étrons. Plus tard, après le dévoilement de son secret et son meurtre, son fils sera traumatisé par les moqueries relatives à une homosexualité héritée de son père. Il déféquera dans la douche de son école, sous les yeux pantois de ses camarades de classe. Le traumatisme de la situation intenable qui engonce les êtres dans l'œuvre, est bien exprimé dans les cas de Vito et de son fils. Ces flatulences et ces congestions évacuées sous une trop forte pression, par des corps qui débordent sous l'effet de forces indicibles, sont l'allégorie du malaise psychologique ambiant.

21 Le personnage de l'agent du FBI est intéressant parce que son malaise a commencé à partir des attentats du 11 septembre 2001, phénomène d'écrasement réel à haute échelle qui vient s'imbriquer au récit fictif. Bourreau quand il faisait chanter Adriana, il devient après le 11 septembre une victime dolente. Lorsque son investigation se concentre sur les signes opaques du terrorisme djihadiste, pour la première fois, nous pouvons discerner une once d'humanité dans la pâleur de son visage et dans la détresse de son regard. Débordé par un combat harassant et sans fin contre un ennemi qu'il est incapable de cerner à partir de sa simple optique opacifiante de policier, oppressé par son supposé statut de super-héros sauveur d'une Amérique en crise civilisationnelle, son ventre pâtit d'un décalage entre phantasme idéal et réalité dégradée : lors de son séjour de lutte antiterroriste au Pakistan, il contracte un virus intestinal qui lui fera perdre l'appétit. Son statut actanciel se métamorphose alors à la $\mathrm{VI}^{\mathrm{e}}$ saison: d'opposant, il devient l'adjuvant qui aide Tony à se débarrasser de son grand ennemi Phil Leotardo. Cette métamorphose sémiotique dit l'aspect fuyant et fluctuant des relations intersubjectives dans le tourbillon desquelles les individus se trouvent absorbés par des forces insoutenables qui les dépassent tragiquement.

\section{Devant le vertige de l'atavisme}

$22 \mathrm{Au}$ dixième épisode de la $\mathrm{V}^{\mathrm{e}}$ saison, ayant rompu avec sa femme Carmela, Tony retourne au domicile conjugal dont son épouse l'avait chassé pour cause de tromperie pour se baigner dans la piscine. Il se retrouve devant une piscine vide, ce qui l'irrite énormément puisque la baignade est l'un de ses divertissements préférés, parce qu'elle constitue sûrement un moment d'apesanteur où son corps s'allège et se confronte doucement aux lois de l'attraction. Sa femme l'informe alors qu'elle l'a vidée parce que la facture devenait trop coûteuse pour une eau que personne n'utilisait. Dès lors, devant la vacuité de la piscine, une pénible scène de déchirement représentée avec théâtralité, commence. Mais dans le positionnement de la caméra, l'angle de prise de vue se détraque soudain en un mouvement descendant. S'immisçant dans un champ/ contrechamp, un plan fixe les cadre en contre-plongée, depuis les profondeurs de la piscine vide, conférant à la scène une inquiétante étrangeté. Les relations intersubjectives apparaissent clairement d'après ce plan furtif sous la forme d'un grand siphonage où chacun se replie dans les abîmes de son ego. Personne n'est capable de comprendre l'autre ou d'interpréter ses inquiétudes. Le dialogue, dans sa féroce quotidienneté, se veut une tyrannique tentative de faire en sorte que l'autre pratique 
ses injonctions et se plie à ses désirs. Il n'est pas une remise en question de sa propre compréhension du monde ainsi que des présupposés qui l'arc-boutent. De ce fait, en vertu de cette contre-plongée, l'échange discursif est un ploiement devant le vertige, une étape de fracture, un acte risqué où l'on pourrait être absorbé par le tourbillon hystérique de l'autre, comme on peut trébucher dans la piscine vide.

Cette contre-plongée fait planer une certaine nocivité psychologique sous-jacente et annonce la tentative de suicide d'Anthony Junior. En effet, à la dernière saison, l'apesanteur de la piscine dans laquelle Tony aimait flotter, devient instrument d'anéantissement pour son fils. Celui-ci animé du désir d'en finir avec la pression d'une foule d'injonctions (parents autoritaires, culte de la virilité, torrent médiatique antiterroriste...) voudra annihiler cette apesanteur en s'attachant à un pavé, avant de plonger dans l'eau. Le pavé touche le fond et Antony lesté se serait noyé si son père n'était pas intervenu de justesse. Le trop-plein de confort présupposé par la piscine laisse place à une concavité funeste dont l'attraction absorbe les êtres fragiles.

La fragilité saturnienne d'Anthony est la manifestation d'une puissante tare familiale : la folie et la dégénérescence cognitive. La mère de Tony ainsi que son oncle Corrado se fourvoient dans le labyrinthe mental de la maladie d'Alzheimer. Mais avant de s'y fourvoyer totalement, ils voudront attirer Tony avec eux dans leur chute. Perdant progressivement toute compétence logique et discursive, ils ne cessent de le dérouter par un crescendo d'hostilité consciente et inconsciente. Sa mère entraînée par une incohérence incontrôlée, fomentera son meurtre. Corrado tirera sur lui et l'enverra dans l'univers sans mémoire du coma. Leur pathologie vertigineuse sera comparée à un trou noir au douzième épisode de la $\mathrm{III}^{\mathrm{e}}$ saison, lorsque Tony excédé par la jalousie belliqueuse de son indomptable maitresse, lui lancera: "Je ne viens pas de te rencontrer. Je t'ai connu toute ma putain de vie. Ma mère était pareille que toi : un putain de trou noir sans fond».

La dégénérescence cognitive semble être une obsession dans la conscience romanesque occidentale des $\mathrm{XIX}^{\mathrm{e}}, \mathrm{Xx}^{\mathrm{e}}$ et $\mathrm{XXI}^{\mathrm{e}}$ siècles. Nous nous bornerons à citer à titre d'exemples des romans tels que Bartleby le Scribe d'Herman Melville qui, outre la thèse deleuzienne qui lui confère une charge de résistance passive, pourrait être l'histoire d'un personnage beckettien avant l'heure qui perd progressivement ses compétences cognitives et pragmatiques jusqu'à sombrer dans un recroquevillement monomaniaque scandé par un leitmotiv dont le sens est incernable: I Would prefer not to; Le Bruit et la Fureur de William Faulkner qui pratique en partie une focalisation interne sur la perception de Benjamin Compson, attardé mental issu d'une lignée lymphatique et maudite. Le lecteur perçoit le monde par le filtre de la subjectivité bégayante d'un idiot. Cette tradition des auteurs attirés par la déficience mentale se poursuivra au Xxi siècle avec ces œuvres que l'on désigne sous le nom de l'Alzheimer literature et dont fait partie The Story of Forgetting de Stefan Merrill Block. Pascale Antolin affirme dans un article consacré à cet ouvrage :

Les publications sur le thème de la maladie, souvent qualifiées de "pathographies " ou même d'« autopathographies", se multiplient des deux côtés de l'Atlantique, depuis une dizaine d'années, au point de constituer une nouvelle catégorie d'œuvres très contemporaines $[. . .]^{33}$

Mais l'œuvre qui nous paraît être proche des Soprano, ne serait-ce déjà que sur le plan d'un patronyme qui constitue le titre d'une œuvre, est l'ensemble des vingt romans composant Les Rougon-Macquart d'Émile Zola. On pourrait très bien détourner le sous- 
titre de cette œuvre, Histoire naturelle et sociale d'une famille sous le Second Empire, pour l'apposer aux Soprano, ce qui donnerait: Histoire naturelle et sociale d'une famille américaine à la fin $d u X X^{e}$ siècle. L'idée d'un souffle héréditaire de folie qui passe outre les générations, en une période libérale de grands chamboulements techniques, anthropologiques et idéologiques, est bien entendu le thème central des deux œuvres sérielles. Pour Zola, il s'agissait de décrire l'arborescence vertigineuse et détraquée d'une famille particulière, à une époque dominée par une "orgie d'appétits et d'ambitions » (Zola 1872). Et c'est dans ce monde nerveux et boulimique que les êtres décrits se consumeront aussi intensément que Les Soprano. Zola indique dans ses notes préparatoires que « les Rougon-Macquart brûleront comme une matière qui se dévore elle-même; ils épuiseront leur corps et leur esprit en trois générations, parce qu'ils auront vécu trop vite ». Pareil destin est réservé à la lignée des Soprano dont les générations s'épuisent dans le rythme épileptique du rêve américain, malgré la conclusion relativement sereine de la dernière saison. Et dans nos deux œuvres, une interrogation importante se maintient à travers les siècles : comment la multitude des possibilités de réussite sociale déséquilibre-t-elle dangereusement la conscience des générations successives, en oblitérant chez elles une indispensable réflexion philosophique, historique et éthique?

Peut-être ce vertige, commençant par la peur de l'autre et finissant dans un regard global pessimiste sur l'évolution de l'usage de la liberté, annonce-t-il, au même titre que la chute du roman de son piédestal et son remplacement par la série, une déconnexion amnésique progressive avec les rêves originels d'« une société libre, pacifique et prospère » (Michéa, 2010,127) de la civilisation libérale.

\section{Conclusion}

De tout ce bruit et de toute cette fureur, quelques idées principales peuvent être dégagées à la fin de cet article : d'abord sur le plan de la production de l'œuvre, des individus en proie à des émotions pathiques et esthétiques rudimentaires s'interrogent gauchement sur le sens d'une succession d'encodages. Les personnages mènent en fait deux quêtes interprétatives: la première est celle de l'interprétation des symboles émergeant de l'abîme, matériel ou immatériel, qui les traverse. Car il faut souligner que le plan initial de l'œuvre, clé de voûte de sa compréhension, montre Tony contemplant avec perplexité une statue posée dans la salle d'attente de sa psychanalyste, d'une manière qui laisse à penser qu'il fait un grand effort pour en comprendre le sens. Si l'on reconnaît avec Jacques Rancière que le point commun entre esthétique et inconscient est la présence de cette «non-pensée qui habite la pensée et lui donne une puissance spécifique $»^{34}$, alors on perçoit la pertinence de ce plan ainsi que d'autres où l'on voit des personnages attirés par des œuvres d'art. Il annonce que l'enjeu principal des Soprano réside dans l'auscultation interprétative de la puissance de cette non-pensée, auscultation que l'ancrage dans une aire inédite dans l'histoire du capitalisme rend féconde. La deuxième est celle du déchiffrement de leur propre Abîme/Chaos/Sans-Fond habité par l'irrationnel et l'infini immatériel. À ce tropisme s'opposent des contraintes matérielles, des injonctions, des dogmes, des déficiences ataviques, des propagandes et des passions.

29 Ensuite, sur le plan de la réception, à travers la mise en évidence du rapport entre la série télé moderne représentée ici par Les Soprano et certains romans-feuilletons, il 
apparaît que la quête est d'autre part celle du récepteur attelé à une œuvre surdéterminée à la fois par des attaches symboliques avec la littérature et l'art ; et par une représentation paradoxale et spectaculaire, immanente aux médias de masse.

De ce fait la production et l'interprétation du symbole moderne, qu'il soit social, onirique ou poïétique, hantent les plis de cette œuvre obèse, si l'on voulait emprunter le mot véhément d'Isidore Isou à propos du cinéma dans Traité de bave et d'éternité; et constituent, en dernière instance, sa contradictoire visée ontologique.

\section{BIBLIOGRAPHIE}

Adorno Theodor W., «L'industrie culturelle », texte des conférences à l'Université radiophonique internationale, Communications, $\mathrm{n}^{\circ}$ 3, 1964, p. 12-18.

Agamben Giorgio, Qu'est-ce que le contemporain ?, traduit de l'italien par Maxime Rovere, Paris, Payot et Rivages, 2008.

Antolin Pascale, «L'écriture thérapeutique de Stefan Merrill Block dans The Story of Forgetting ", Transatlantica, $\mathrm{n}^{\circ}$ 1, 2013, http://transatlantica.revues.org/6410

Arrivé Michel, Le linguiste et l'inconscient, Paris, PUF, 2008.

Aubry Danielle, Du roman-feuilleton à la série télévisuelle. Pour une rhétorique du genre et de la sérialité, Berne, Peter Lang, 2006.

Balzac Honoré de, Illusions Perdues, version numérique, http://gallica.bnf.fr/, 1837.

Barthes, Roland, S/Z, Paris, Seuil, coll. « Points », [1970], 1976.

Bautier Roger, Cazenave Élisabeth, « La presse pousse-au-crime selon Tarde et ses contemporains ", Champ pénal / Penal field, XXXIV Congrès français de criminologie, «Les criminologiques de Tarde », 2008, http://champpenal.revues.org/253, DOI : 10.4000/champpenal. 253

Castoriadis Cornelius, Figures du pensable VI, Paris, Seuil, 1999.

Cornelius Castoriadis, L'institution imaginaire de la société, Paris, Seuil, [1975], 1999.

Compagnon Antoine, Les cinq paradoxes de la modernité, Paris, Seuil, 1990.

Debord Guy, La société du spectacle, Paris, Folio Gallimard, [1967], 1992.

Esquenazi Jean-Pierre, Les Séries télévisées, L'avenir du cinéma ?, Paris, Armand Colin, 2010.

Faulkner William, Le Bruit et la Fureur, Paris, Gallimard, [1949], 2003.

Foubert Frédéric et Loulendo Florent, Les Soprano. Portrait d'une Amérique désenchantée, Paris, Éditions Cathodique, 2009.

Gautier Théophile, Mademoiselle de Maupin, Version numérique, http://gallica.bnf.fr/, 1876.

Genette Gérard, Figures III, Paris, Seuil, coll. « Poétique », 1972.

Goldmann Lucien, Pour une sociologie du roman, Paris, Gallimard, coll. « Tel », [1964], 1986. 
Goudmand Anaïs, « Narratologie du récit sériel », Proteus, n 6, 2013, p. 81-90, http://www.revueproteus.com/articles/Proteus06-10.pdf.

Hauser Arnold, Histoire sociale de l'art et de la littérature, Paris, PUF, coll. « Quadrige », [1982], 2004.

Jauss Hans Robert, Pour une esthétique de la réception, traduit de l'allemand par Claude Maillard, Paris, Gallimard, [1978], 1991.

Jost François, De quoi les séries américaines sont-elles le symptôme ?, Paris, CNRS édition, 2011.

Melville Herman, Bartleby le scribe, Paris, Gallimard, [1853], 2001.

Meyer Michel, Le comique et le tragique. Penser le théâtre autrement, Paris, PUF, 2003.

Michéa Jean-Claude, L'empire du moindre mal. Essai sur la civilisation libérale, Paris, Flammarion, 2010.

Murata Kyoko, «Assimilation de l'esthétique du roman-feuilleton chez Balzac », in Balzac et alii, génétiques croisées. Histoires d'éditions, Takayuki Kamada et Jacques Neefs (dir.), Colloque international organisé par le GIRB les 3-5 juin, 2010, http://balzac.cerilac.univ-paris-diderot.fr/ wa_files/Murata.pdf

Rancière Jacques, L'Inconscient esthétique, Paris, Galilée, [2001], 2009.

Richard Jean-Pierre, Littérature et Sensation, Paris, Seuil, 1954.

Tarde Gabriel, L'opinion et la Foule, Paris, Felix Alcan, 1901.

Tocqueville Alexis de, De la démocratie en Amérique, Paris, Flammarion, [1835], 1981.

Winock Michel, Flaubert, Paris, Gallimard, 2013.

Wunenburger Jean-Jacques, « Mirages de l'image à l'ère électronique », in Les Dons de l'Image, Alain Cambier (dir.), Paris, L'Harmattan, 2003.

Zola Émile, CEuvres. Manuscrits et dossiers préparatoires. Les Rougon-Macquart. Notes préparatoires à la série des Rougon-Macquart, Bibliothèque nationale de France, département des Manuscrits, 1872, http://gallica.bnf.fr

Zola Émile, L'assommoir, Paris, Flammarion, [1876], 2000.

\section{NOTES}

1. Gérard Genette, Figures III, Paris, Seuil, coll. « Poétique », 1972.

2. Comme le dira souvent le personnage Christopher Moltisanti et comme le suggérera la métaphore filée de la stratégie militaire. Cette référence tend à installer un élancement guerrier à une organisation criminelle qui a tendance à se désagréger dans les différents égos infantiles, démesurés et conflictuels qui la composent.

3. Son univers, englobant des personnages variés et antagonistes qui s'y entrechoquent, prend une consistance figurative devant sa psychanalyste, dès que sa voix-off commence à rapporter les événements qu'il a vécu pendant la journée où il s'est évanoui. Le déploiement de la narration, qui commence par un gros plan sur l'œil de Tony connotant une sorte d'éveil à la diégèse, est donc fondamentalement joint à l'herméneutique psychanalytique.

4. Celle-ci conçoit la thérapie comme un accouchement tandis que Tony la conçoit plutôt comme une défécation. Dans les deux cas, l'origine de l'énonciation thérapeutique est le ventre.

5. Roland Barthes, S/Z, Paris, Seuil, coll. « Points », [1970], 1976. 
6. La grande spécialité de Tony est le barbecue de saucisses, ce qui n'est pas innocent dans ce contexte.

7. L'autonomie, selon Castoriadis, est la phase qu'atteignent dialectiquement les individus et la société, en conséquence d'un effort de réflexion permanente de soi-même sur soi-même ainsi que sur toutes les institutions sociales. Par institutions sociales, il entend des domaines comme le langage, la religion ou le pouvoir... La remise en cause philosophique et politique des institutions sociales a pour objectif à la fois d'empêcher les tendances individuelles et collectives de se soumettre à des lois extra-sociales aliénantes comme le dogme religieux ou le novlangue linguistique et de remettre sans cesse la société au centre producteur des normes institutionnelles. Le discours proféré face au psychanalyste en l'occurrence est une condition nécessaire de cette autonomie parce qu'il permet d'établir une subjectivité réflexive et délibérative.

8. David Cronenberg est un réalisateur qui semble être obsédé par l'exploration anatomique sanglante du corps humain. Dans des œuvres fantastiques telles que Vidéodrome ou Existenz, il donne l'impression d'être un aruspice postmoderne fouillant dans les entrailles de ses personnages pour essayer de donner un sens à la fuite en avant spectaculaire et technologisée de la civilisation humaine.

9. L'allégorie, par son travail de représentation de l'abstraction, est un agencement antinomique de matériel et d'immatériel. Dans ce sens l'allégorie est, si l'on veut, la figure de style idéale de notre œuvre où est constamment sondé l'indicible souffle qui hante l'anatomie humaine.

10. Cornelius Castoriadis, Figures du pensable VI, Paris, Seuil, 1999, p. 101.

11. Jean-Pierre Richard, Littérature et Sensation, Paris, Seuil, 1954, p. 119.

12. Jacques Rancière, L'Inconscient esthétique, Paris, Galilée, [2001], 2009, p. 12.

13. Jean-Pierre Richard, op. cit., p. 122.

14. Jean-Claude Michéa, L'empire du moindre mal. Essai sur la civilisation libérale, Paris, Flammarion, 2010.

15. Ce concept s'inspire à la fois des travaux de Georg Lukács sur le roman et de la théorie de la médiatisation de René Girard.

16. Lucien Goldmann, Pour une sociologie du roman, Paris, Gallimard, coll. « Tel », [1964], 1986, p. 49.

17. Voir la critique de l'historicisme de Goldmann et de Lukács (Hans Robert Jauss, Pour une esthétique de la réception, traduit de l'allemand par Claude Maillard, Paris, Gallimard, [1978], 1991, p. 36-38). Nous renvoyons également à Figures III et à l'« insatisfaction » de Gérard Genette vis-àvis de la méthode sociocritique. (Genette, op. cit., p. 16-18).

18. Voir la préface sarcastique de son roman Mademoiselle de Maupin.

19. Voir son roman Illusions Perdues.

20. Dans son ouvrage L'opinion et la Foule, Tarde conçoit la différence entre livre et journal de manière peu flatteuse pour ce dernier : «L'influence du livre, qui a précédé celle du journal, et qui au XVIII ${ }^{\mathrm{e}}$ siècle comme au XVII ${ }^{\mathrm{e}}$ a été dominante, ne pouvait produire les mêmes effets; car si le livre faisait sentir aussi à tous ceux qui le lisaient dans la même langue leur identité philologique, il ne s'agissait plus là de questions actuelles et simultanément passionnantes pour tous. L'existence nationale est bien attestée par les littératures, mais ce sont les journaux qui attisent la vie nationale, qui soulèvent les mouvements d'ensemble des esprits et des volontés en leurs quotidiennes fluctuations grandioses. Au lieu de puiser son intérêt propre, comme le journal, dans l'actualité concrète de ces informations, le livre cherche à intéresser avant tout par le caractère général et abstrait des idées qu'il apporte. Il est donc plus apte à susciter un courant humanitaire, comme l'a fait notre littérature du XvIII ${ }^{\mathrm{e}}$ siècle, qu'un courant national ou même international. Car international et humanitaire font deux : une fédération européenne, telle que nos internationalistes peuvent s'en faire une notion très positive, n'a rien de commun avec «l'humanité » divinisée par les encyclopédistes, dont Auguste Comte a dogmatisé les idées sur ce 
point. Par suite, il y a lieu de penser qu'à la prépondérance du livre sur le journal comme éducateur de l'opinion tient le caractère cosmopolite et abstrait des tendances de l'esprit public au moment où s'est ouverte la Révolution de 1789 » (Gabriel Tarde, L'opinion et la Foule, Paris, Felix Alcan, 1901, p. 81-82).

21. Le film Vidéodrome de David Cronenberg (1983) est très pessimiste quant à la question de la prédominance voyeuriste de l'image télévisée. Ce pessimisme va jusqu'à percevoir dans cette image un instrument de mort. Mais il nous semble qu'il y a un paradoxe gênant dans toute critique du spectacle par le biais du spectacle, paradoxe irrésolu même dans certains essais visuels des plus efficients critiques de l'image tels que Guy Debord ou Isidore Isou.

22. À cette exception près qu'il s'agit de deux canaux de communication différents : l'écriture pour la presse et l'image pour la télévision.

23. Roger Bautier et Élisabeth Cazenave, «La presse pousse-au-crime selon Tarde et ses contemporains", Champ pénal/ Penal field, XXXIV Congrès français de criminologie, «Les criminologiques de Tarde », 2008.

24. Une étude narratologique d'Anaïs Goudmand sur le récit sériel décèle un effet marketing de «fidélisation sur le long terme» instauré par la série télé et le roman-feuilleton chez le récepteur.

25. Arnold Hauser, Histoire sociale de l'art et de la littérature, Paris, PUF, coll. « Quadrige », [1982], 2004, p. 642.

26. Antoine Compagnon, Les cinq paradoxes de la modernité, Paris, Seuil, 1990, p. 20.

27. Theodor W. Adorno, "L'industrie culturelle», texte des conférences à l'Université radiophonique internationale, Communications, $\mathrm{n}^{\circ} 3,1964$, p. 12-18.

28. Jean-Jacques Wunenburger émet de son côté une interrogation véhémente mais qui n'en contient pas moins des termes révélateurs sur le financement du type de productions dont fait partie notre œuvre :

«[...] on observe une sorte de silence servile face à ce capital du spectacle et du loisir, qui tranche avec la critique adressée aux marchands d'armes, aux entreprises de biens de productions et de services. Ni le salaire d'acteurs, ni le coût d'un film n'inspirent de réflexion systématique sur le gaspillage de richesses. N'est-ce pas le signe d'une sorte d'idolâtrie planétaire, l'image étant un Dieu qui vaut tous les sacrifices?" (Jean-Jacques Wunenburger, "Mirages de l'image à l'ère électronique », in Les Dons de l'Image, Alain Cambier (dir.), Paris, L'Harmattan, 2003, p. 97).

Les termes « idolâtrie », « Dieu » et « sacrifice " par lesquels se termine la citation renvoient à un champ lexical du sacré. Et ce champ lexical fait référence d'un côté à une sorte de sacralisation de l'image, à une absence d'esprit critique devant sa fabrication de plus en plus opulente, et d'un autre côté aux origines religieuses du théâtre tragique grec où il était question du sacrifice de bouc-émissaires. On peut donc dire qu'il s'interroge implicitement sur les conditions poiétiques, économiques et anthropologiques en vertu desquelles l'humanité est passée du sacré consubstantiel au théâtre tragique pendant l'Antiquité grecque, à la trivialité commerciale des œuvres diffusées par un écran contemporain.

29. Giorgio Agamben, Qu'est-ce que le contemporain ?, traduit de l'italien par Maxime Rovere, Paris, Payot et Rivages, 2008, p. 21.

30. Alexis de Tocqueville, De la démocratie en Amérique, Paris, Flammarion, [1835], 1981, p. 273.

31. Michel Meyer, Le comique et le tragique. Penser le théâtre autrement, Paris, PUF, 2003.

32. L'interaction entre sémiotique et interprétation du rêve, que nous décelons dans notre l'œuvre, a été évoquée dans les sciences humaines, entre autres par Michel Arrivé : «[...] le rêve est un objet sémiotique. C'est l'opinion de Freud. Il n'utilise certes pas l'adjectif "sémiotique" : il ne l'avait pas à sa disposition, pour des raisons historiques du même type que celles qui l'empêchaient d'alléguer explicitement "métalangage" ou “autonymie". Mais l'ensemble des analyses de la Traumdeutung - le rêve comme texte, le rêve comme écriture, le rêve comme traduction des pensées latentes en une autre langue - est à l'évidence l'exact équivalent d'une 
définition sémiotique du rêve. Le rêve pour Freud est un discours, et par là relève d'un langage » (Michel Arrivé, Le linguiste et l'inconscient, Paris, PUF, 2008, p. 75).

33. Pascale Antolin, «L'écriture thérapeutique de Stefan Merrill Block dans The Story of Forgetting », Transatlantica, $\mathrm{n}^{\circ} 1,2013$.

34. Jacques Rancière, op. cit., p. 33.

\section{RÉSUMÉS}

Les prétentions artistiques de la série télé Les Soprano nous ont convaincu qu'elle s'inscrit dans une période nouvelle de l'histoire de la culture de masse, avec tout ce que cela implique de transformations esthétiques fondamentales. Nous mettrons cette période en parallèle avec celle des origines du genre romanesque et de l'apparition du roman-feuilleton, à travers le concept de l'individu problématique de Lucien Goldmann, enrichi par le travail de Jean-Pierre Richard sur la littérature de Flaubert ainsi que la théorie de la réception de Hans Robert Jauss. Nous ferons dans ce sens s'interpénétrer esthétique littéraire, narratologie, analyse filmique, histoire et sociologie afin de montrer que l'œuvre est habitée, de manière aussi bien volontaire qu'involontaire, par certaines contradictions multidimensionnelles, inhérentes aux mutations de la civilisation libérale et que l'Abîme/Chaos/Sans-Fond de Cornelius Castoriadis, par sa richesse conceptuelle, peut contribuer à décrire.

\section{INDEX}

Mots-clés : série télé, esthétique, sémiotique, image, roman-feuilleton, libéralisme, individualisme, médias, télévision, interprétation, art, littérature, spectacle, industrie culturelle, digestion, journalisme, nourriture, Abîme/Chaos/Sans-Fond, vide, Soprano, individu problématique

Personnes citées : Castoriadis, Tarde, Zola, Michéa, Genette, Goldmann

\section{AUTEUR}

\section{AYMEN GHARBI}

Chercheur en littérature à l'université Paris 8, aymengharbi68@yahoo.fr 